Bull. Korean Math. Soc. 50 (2013), No. 4, pp. 1099-1108

http://dx.doi.org/10.4134/BKMS.2013.50.4.1099

\title{
MANNHEIM CURVES IN 3-DIMENSIONAL SPACE FORMS
}

\author{
Jin Ho Choi, Tae Ho Kang, and Young Ho Kim
}

\begin{abstract}
We define the Mannheim curve in a 3-dimensional Riemannian manifold, which is a generalization of the Mannheim curve in Euclidean space. In particular, we study the Mannheim curves and their partner curves in 3-dimensional space forms.
\end{abstract}

\section{Introduction}

In a classical differential geometry, the properties of curves in a 3-dimensional Euclidean or Minkowski space are generally characterized by the algebraic equations concerning their curvature and torsion function. For example, the general helices and the Bertrand curves in a 3-dimensional Euclidean space $\mathbb{E}^{3}$ are characterized respectively by

$$
a \kappa-\tau=0 \text { and } a \kappa+b \tau=1
$$

for some constants $a \neq 0$ and $b$, where $\kappa$ and $\tau$ are the curvature function and the torsion function under consideration.

On the other hand, it is well-known that the Mannheim curves in $\mathbb{E}^{3}$ are characterized by

$$
\kappa=a\left(\kappa^{2}+\tau^{2}\right)
$$

for a constant $a \neq 0$. Moreover, H. Liu and F. Wang proved that Mannheim partner curves in $\mathbb{E}^{3}$ are characterized by

$$
\kappa^{\prime}=\frac{\kappa}{a}\left(1+a^{2} \tau^{2}\right)
$$

where ' denotes the differentiation with respect to the arc length parameter of a given curve (see, [2]).

Recently, the authors defined the Bertrand curves in a 3-dimensional Riemannian manifold and proved that those in 3-dimensional space forms are characterized by the same algebraic equation as that in $\mathbb{E}^{3}$ (see, [1]).

Received March 30, 2012; Revised October 25, 2012.

2010 Mathematics Subject Classification. Primary 53A04, 53A35.

Key words and phrases. Mannheim curves, Mannheim partner curves, space form.

The third author was supported by Basic Science Research Program through the National Research Foundation of Korea (NRF) funded by the Ministry of Education, Science and Technology (2010-0007184). 
In this paper, we define the Mannheim curves in a 3-dimensional Riemannian manifold and give the algebraic characterizations of Mannheim curves and their partner curves in 3-dimensional space forms.

\section{Preliminaries and definitions}

Let $M:=(M,\langle\rangle$,$) be a 3$-dimensional Riemannian manifold and $\nabla$ denotes the Levi-Civita connection of $M$. Let $T_{p} M$ denotes the set of all tangent vectors to $M$ at $p \in M$. For a vector $v$ in $T_{p} M$, we define the norm of $v$ by $\|v\|:=\sqrt{\langle v, v\rangle}$.

Let $\alpha: I \rightarrow M$ be a smooth curve in $M$. If $\left\|\alpha^{\prime}(s)\right\|=1$ for each $s \in I$, the curve $\alpha$ is called a unit speed curve. In this case, the parameter $s$ can be assumed to be the arc length parameter of $\alpha$.

A vector field $X$ on $M$ along $\alpha$ is said to be parallel along $\alpha$ if $\nabla_{s} X=0$ for all $s$, where $\nabla_{s}$ denotes the covariant derivative along $\alpha$. When a vector field $X$ on $M$ is parallel along $\alpha$, a vector $X_{\alpha\left(s_{1}\right)}$ at $\alpha\left(s_{1}\right)$ is called the parallel displacement of a vector $X_{\alpha\left(s_{0}\right)}$ at $\alpha\left(s_{0}\right)$ along $\alpha$.

A smooth curve $\alpha: I \rightarrow M$ is called a geodesic in $M$ if its velocity vector field $\alpha^{\prime}$ is parallel along $\alpha$. A non-geodesic unit speed smooth curve $\alpha$ is called a Frenet curve on $M$ if there exists an orthonormal frame $\left\{T=\alpha^{\prime}, N, B\right\}$ along $\alpha$ and smooth functions $\kappa_{\alpha}>0$ and $\tau_{\alpha}$ satisfying the following system of ordinary differential equations

$$
\nabla_{s}\left(\begin{array}{c}
T \\
N \\
B
\end{array}\right)=\left(\begin{array}{ccc}
0 & \kappa_{\alpha} & 0 \\
-\kappa_{\alpha} & 0 & \tau_{\alpha} \\
0 & -\tau_{\alpha} & 0
\end{array}\right)\left(\begin{array}{l}
T \\
N \\
B
\end{array}\right)
$$

The equation (2.1) is called the Frenet formula of the Frenet curve $\alpha$. The functions $\kappa_{\alpha}, \tau_{\alpha}$ and the orthonormal frame $\{T, N, B\}$ are called the curvature, the torsion and the Frenet frame of $\alpha$, respectively. It is well-known that the curvature and the torsion are invariant under the isometries of $M$. Three unit vector fields $T, N$ and $B$ consisting of the Frenet frame of $\alpha$ are called the tangent, principal normal and binormal vector field, respectively.

Denote the exponential map at $p \in M$ by $\exp _{p}$. Recall that the exponential map $\exp _{p}: T_{p} M \rightarrow M$ at $p \in M$ is defined by

$$
\exp _{p}(v)=\gamma_{v}(1)
$$

where $\gamma_{v}:[0, \infty) \rightarrow M$ is the constant speed geodesic starting from $p$ with the initial velocity $\gamma_{v}(0)=v$.

Now, we define a Mannheim curve in a 3-dimensional Riemannian manifold $M$ as follows:

Definition 2.1. Let $\alpha(s)$ be a Frenet curve in a 3-dimensional Riemannian manifold $M$ and $\left\{T_{\alpha}, N_{\alpha}, B_{\alpha}\right\}$ the Frenet frame of $\alpha$. Consider a surface $X_{N_{\alpha}}$ defined by

$$
X_{N_{\alpha}}(s, t)=\exp _{\alpha(s)}\left(t N_{\alpha}(s)\right) .
$$


A Frenet curve $\beta=\beta(s)$ in $M$ is called a Mannheim partner curve of $\alpha$ if the binormal vector field of $\beta$ defined by $\beta(s)=X_{N_{\alpha}}(s, t(s))$ is the parallel displacement of $N_{\alpha}\left(s_{0}\right)$ or $-N_{\alpha}\left(s_{0}\right)$ for each $s=s_{0}$. When a Frenet curve $\alpha$ in $M$ admits its Mannheim partner curve, we call $\alpha$ a Mannheim curve in $M$.

Definition 2.1 is trivially equivalent to the following:

Definition 2.2. For two different Frenet curves $\alpha(s)$ and $\beta(s)$ on $M, \beta$ is a Mannheim partner curve of $\alpha$ if $\alpha(s)=X_{B_{\beta}}(s, t(s))$ and for each $s=s_{0}$, its principal normal vector $N_{\alpha}$ at $\alpha\left(s_{0}\right)$ is the parallel displacement of $B_{\beta}$ or $-B_{\beta}$ at $\beta\left(s_{0}\right)$ along the ruling curve at $\beta\left(s_{0}\right)$. When a Frenet curve $\alpha$ in $M$ admits its Mannheim partner curve, we call $\alpha$ a Mannheim curve on $M$.

Remark 2.1. Generally, a straight line $\ell$ in a 3 -dimensional Euclidean space $\mathbb{E}^{3}$ can not define its Frenet frame. But, in the study of Bertrand curves and Mannheim curves, the straight line $\ell$ is regarded as a Frenet curve with arbitrary Frenet frame $\{T, N, B\}$ for a unit tangent vector $T$ of $\ell$. In this paper, we consider a geodesic in a 3-dimensional manifold $M$ as a Frenet curve with a Frenet frame $\{T, N, B\}$ by choosing $N$ and $B$ properly, where $T$ is the unit tangent vector field $T$ of the geodesic.

\section{Mannheim curves in 3-dimensional space forms}

Let $M$ be a 3 -dimensional simply connected space form, i.e., $\mathbb{E}^{3}, \mathbb{S}^{3}$ or $\mathbb{H}^{3}$, and $\nabla$ the Levi-Civita connection of $M$. Here, we consider $M$ as a subspace of $\mathbb{E}^{4}$ with the induced metric from the natural inner product $\langle$,$\rangle of \mathbb{E}^{4}$ (resp., $\mathbb{E}_{1}^{4}$ ) if $M=\mathbb{E}^{3}$ or $\mathbb{S}^{3}:=\left\{p \in \mathbb{E}^{4} \mid\langle p, p\rangle=1\right\}$ (resp., $\mathbb{H}^{3}:=\left\{p \in \mathbb{E}_{1}^{4} \mid\langle p, p\rangle=-1\right\}$ ).

Let $\alpha$ be a smooth curve in $M$ and ' denotes the ordinary differentiation with respect to the parameter of $\alpha$ in $\mathbb{E}^{4}$ or $\mathbb{E}_{1}^{4}$. Then, the Gauss formula of $M$ along $\alpha$ is given by

$$
X^{\prime}=\nabla_{s} X-\epsilon\left\langle X, \alpha^{\prime}\right\rangle \alpha
$$

for any vector field $X$ on $M$ along $\alpha$, where $\nabla_{s}$ denotes the covariant derivative of $M$ along the curve $\alpha$ and $\epsilon=-1,0$ or 1 if $M$ is $\mathbb{H}^{3}, \mathbb{E}^{3}$ or $\mathbb{S}^{3}$, respectively.

The exponential map $\exp _{p}(t v)$ on $M$ and the parallel transport $P^{t}(v)$ from $p$ to $\exp _{p}(t v)$ along the geodesic $\gamma_{v}$ are well-known as the following simple expressions (see, [1]):

$$
\exp _{p}(t v)=f(t) p+g(t) v
$$

and

$$
P^{t}(v)=-\epsilon g(t) p+f(t) v
$$


where $p \in M$ and $v \in T_{p} M$ with $\|v\|=1$ are considered as vectors in $\mathbb{E}^{4}$ and the functions $f$ and $g$ are given by

$$
\begin{cases}f(t)=1 & g(t)=t, \quad \text { if } M=\mathbb{E}^{3}, \\ f(t)=\cos t & g(t)=\sin t, \quad \text { if } M=\mathbb{S}^{3}, \\ f(t)=\cosh t & g(t)=\sinh t, \quad \text { if } M=\mathbb{H}^{3}\end{cases}
$$

and $\epsilon=-1,0$ or 1 if $M$ is $\mathbb{H}^{3}, \mathbb{E}^{3}$ or $\mathbb{S}^{3}$, respectively.

Now, we will consider the characterizations with respect to the curvature and the torsion of Mannheim curves in 3-dimensional space forms.

In case of $M=\mathbb{E}^{3}$, the following proposition is well-known.

Proposition 3.1. A Frenet curve $\alpha$ in $\mathbb{E}^{3}$ with the curvature $\kappa$ and the torsion $\tau$ is a Mannheim curve if and only if it satisfies $\kappa=a\left(\kappa^{2}+\tau^{2}\right)$ for a constant $a \neq 0$.

Let $M$ be a 3 -dimensional non-flat space form, i.e., $\mathbb{S}^{3}$ or $\mathbb{H}^{3}$, and let $\alpha=\alpha(s)$ be a unit speed Frenet curve in $M$ satisfying (2.1) and $\beta=\beta(u)$ a Mannheim partner curve of $\alpha$, where $u$ is the arc length parameter of $\beta$. Note that we can assume $d u / d s>0$. Then, without loss of generality, the curve $\beta(u)$ and its binormal vector field $B_{\beta}(u)$ can be expressed by

$$
\begin{aligned}
\beta(u) & =\exp _{\alpha(u)}\left(t(u) N_{\alpha}(u)\right) \\
& =f(t(u)) \alpha(u)+g(t(u)) N_{\alpha}(u)
\end{aligned}
$$

and $B_{\beta}(u)= \pm P^{t(u)}\left(N_{\alpha}(u)\right)$, respectively, where the functions $f$ and $g$ are given in (3.4).

By differentiating $\beta$ with respect $u$ as a vector valued function in $\mathbb{E}^{4}$, we have

$$
\begin{aligned}
\beta^{\prime}(u)=\{ & f(t(u))\}^{\prime} \alpha(u)+f(t(u)) \frac{d s}{d u} T_{\alpha}(u) \\
& +\{g(t(u))\}^{\prime} N_{\alpha}(u)+g(t(u)) \frac{d s}{d u} \nabla_{s} N_{\alpha}(u) \\
= & \{f(t(u))\}^{\prime} \alpha(u)+\frac{d s}{d u}\left\{f(t(u))-\kappa_{\alpha}(u) g(t(u))\right\} T_{\alpha}(u) \\
& +\{g(t(u))\}^{\prime} N_{\alpha}(u)+\tau_{\alpha}(u) g(t(u)) \frac{d s}{d u} B_{\alpha}(u) .
\end{aligned}
$$

Since $\left\langle\beta^{\prime}, \beta\right\rangle=\left\langle\beta^{\prime}, B_{\beta}\right\rangle=0$ and $B_{\beta}=-\epsilon g(t) \alpha+f(t) N_{\alpha}$, (3.5) shows that $\beta^{\prime}$ is orthogonal to $\alpha$ and $N_{\alpha}$ in $\mathbb{E}^{4}$ or $\mathbb{E}_{1}^{4}$. Thus, from (3.6), we get

$$
\{f(t(u))\}^{\prime}=\{g(t(u))\}^{\prime}=0 .
$$

Since $t$ is a non-zero smooth function, $t(u)$ is a non-zero constant, say $t(u)=$ $\theta \neq 0$. Then, (3.5) and (3.6) are respectively reduced to

$$
\beta(u)=f(\theta) \alpha(u)+g(\theta) N_{\alpha}(u)
$$


and

$$
\beta^{\prime}(u)=\frac{d s}{d u}\left[\left\{f(\theta)-\kappa_{\alpha}(u) g(\theta)\right\} T_{\alpha}(u)+\tau_{\alpha}(u) g(\theta) B_{\alpha}(u)\right],
$$

from which,

$$
d u / d s=\sqrt{\left\{f(\theta)-\kappa_{\alpha}(u) g(\theta)\right\}^{2}+\tau_{\alpha}(u)^{2} g(\theta)^{2}} .
$$

Note that if $M=\mathbb{S}^{3}$ and $\theta=\pi$ in (3.8), then $\beta=-\alpha$. Also, we can assume $g(\theta) \neq 0$.

For the sake of simplicity, we put

$$
T_{\beta}(u)=a_{1}(u) T_{\alpha}(u)+a_{2}(u) B_{\alpha}(u),
$$

where $a_{1}(u)=\frac{d s}{d u}\left\{f(\theta)-\kappa_{\alpha}(u) g(\theta)\right\}, a_{2}(u)=\frac{d s}{d u} \tau_{\alpha}(u) g(\theta)$.

Differentiating (3.11), we have

$$
\begin{aligned}
\nabla_{u} T_{\beta}(u)-\epsilon \beta(u)= & -\epsilon a_{1}(u) \frac{d s}{d u} \alpha(u)+a_{1}^{\prime}(u) T_{\alpha}(u) \\
& +\frac{d s}{d u}\left\{a_{1}(u) \kappa_{\alpha}(u)-a_{2}(u) \tau_{\alpha}(u)\right\} N_{\alpha}(u)+a_{2}^{\prime}(u) B_{\alpha}(u),
\end{aligned}
$$

from which,

$$
\begin{aligned}
\nabla_{u} T_{\beta}(u)= & \epsilon\left\{f(\theta)-a_{1}(u) \frac{d s}{d u}\right\} \alpha(u)+a_{1}^{\prime}(u) T_{\alpha}(u) \\
& +\left\{a_{1}(u) \kappa_{\alpha}(u) \frac{d s}{d u}-a_{2}(u) \tau_{\alpha}(u) \frac{d s}{d u}+\epsilon g(\theta)\right\} N_{\alpha}(u) \\
& +a_{2}^{\prime}(u) B_{\alpha}(u) .
\end{aligned}
$$

Since $\left\langle N_{\beta}, \beta\right\rangle=\left\langle N_{\beta}, B_{\beta}\right\rangle=0$ and $B_{\beta}=-\epsilon g(t) \alpha+f(t) N_{\alpha}$, (3.5) implies that $N_{\beta}$ is orthogonal to $\alpha$ and $N_{\alpha}$ in $\mathbb{E}^{4}$ or $\mathbb{E}_{1}^{4}$. Thus, from (3.12), we have

$$
\left\{\begin{array}{l}
f(\theta)-a_{1} \frac{d s}{d u}=0 \\
a_{1} \kappa_{\alpha} \frac{d s}{d u}-a_{2} \tau_{\alpha} \frac{d s}{d u}+\epsilon g(\theta)=0
\end{array}\right.
$$

and

$$
\nabla_{u} T_{\beta}=a_{1}^{\prime} T_{\alpha}+a_{2}^{\prime} B_{\alpha}
$$

Then, we get

$$
a_{1}=f(\theta) \frac{d u}{d s} \text { and } a_{2}=\frac{1}{\tau_{\alpha}}\left(f(\theta) \kappa_{\alpha}+\epsilon g(\theta)\right) \frac{d u}{d s},
$$

or equivalently,

$$
\begin{aligned}
f(\theta) & =\frac{f(\theta)-g(\theta) \kappa_{\alpha}}{\left(f(\theta)-g(\theta) \kappa_{\alpha}\right)^{2}+g(\theta)^{2} \tau_{\alpha}{ }^{2}}, \\
f(\theta) \kappa_{\alpha}+\epsilon g(\theta) & =\frac{g(\theta) \tau_{\alpha}{ }^{2}}{\left(f(\theta)-g(\theta) \kappa_{\alpha}\right)^{2}+g(\theta)^{2} \tau_{\alpha}{ }^{2}} .
\end{aligned}
$$


It follows that

$$
\frac{f(\theta)}{f(\theta) \kappa_{\alpha}+\epsilon g(\theta)}=\frac{f(\theta)-g(\theta) \kappa_{\alpha}}{g(\theta) \tau_{\alpha}^{2}},
$$

or, equivalently,

$$
\kappa_{\alpha}^{2}+\tau_{\alpha}^{2}=\lambda \kappa_{\alpha}+\epsilon,
$$

where $\lambda$ is a constant given by

$$
\lambda=\frac{f(\theta)^{2}-\epsilon g(\theta)^{2}}{f(\theta) g(\theta)} .
$$

Note that $\lambda=\theta(\epsilon=0), 2 \cot 2 \theta(\epsilon=1)$ or $\lambda=2 \operatorname{coth} 2 \theta,|\lambda|>2(\epsilon=-1)$.

Conversely, for a curve $\alpha$ in $M$ satisfying (3.18) for some $\lambda(\lambda \neq 0$ if $\epsilon=1$, $|\lambda|>2$ if $\epsilon=-1)$, we may choose $\theta \in \mathbb{R}$ such that $\lambda=\frac{f(\theta)^{2}-\epsilon g(\theta)^{2}}{f(\theta) g(\theta)}$, and hence it satisfies (3.17). Equivalently, (3.17) can be rewritten as

$$
f(\theta)-g(\theta) \kappa_{\alpha}=\frac{f(\theta) g(\theta) \tau_{\alpha}^{2}}{f(\theta) \kappa_{\alpha}+\epsilon g(\theta)} .
$$

We define a curve $\beta$ by (3.8). Since either $f(\theta)=\cos \theta$ and $g(\theta)=\sin \theta$, or $f(\theta)=\cosh \theta$ and $g(\theta)=\sinh \theta$, we can check from (3.19) that two equations of (3.16) are satisfied. Note that (3.16) derives the system of equations of (3.13). Thus, we get from (3.12) the principal normal vector field $N_{\beta}$ of $\beta$ given by

$$
N_{\beta}=\frac{a_{1}^{\prime}}{\sqrt{a_{1}^{\prime 2}+a_{2}^{\prime 2}}} T_{\alpha}+\frac{a_{2}^{\prime}}{\sqrt{a_{1}^{\prime 2}+a_{2}^{\prime 2}}} B_{\alpha} .
$$

Since $\left\{\beta, T_{\beta}, N_{\beta}, B_{\beta}\right\}$ forms an orthonormal frame in $\mathbb{E}^{4}$ or $\mathbb{E}_{1}^{4},(3.8),(3.11)$ and (3.20) yield the binormal vector field $B_{\beta}$ as

$$
B_{\beta}(u)= \pm\left\{-\epsilon g(\theta) \alpha(u)+f(\theta) N_{\alpha}(u)\right\}= \pm P^{\theta}\left(N_{\alpha}\right) .
$$

Therefore, $\beta$ is a Mannheim partner curve of $\alpha$, i.e., $\alpha$ is a Mannheim curve in $M$.

Note that if $f(\theta)=1, g(\theta)=\theta$ and $\epsilon=0,(3.18)$ is replaced by

$$
\kappa_{\alpha}=\frac{1}{\theta}\left(\kappa_{\alpha}^{2}+\tau_{\alpha}^{2}\right) .
$$

Thus, we have an extended characterization of Mannheim curves on a 3dimensional space form.

Theorem 3.2. A Frenet curve $\alpha$ on a 3-dimensional space form $M$ with the curvature $\kappa$ and $\tau$ is a Mannheim curve if and only if it satisfies (3.18) for a constant $\lambda\left(\lambda \neq 0\right.$ if $M=\mathbb{E}^{3}$ or $\mathbb{S}^{3},|\lambda|>2$ if $\left.M=\mathbb{H}^{3}\right)$ with (3.4). 


\section{Mannheim partner curves in 3-dimensional space forms}

In 2008, H. Liu and W. Fang defined the notion of Mannheim partner curve and they proved the following:

Proposition 4.1. Let $\beta=\beta(s)$ be a Frenet curve in $\mathbb{E}^{3}$ with the arc length parameter s. Then $\beta$ is the Mannheim partner curve of some Mannheim curve $\alpha$ in $\mathbb{E}^{3}$ if and only if the curvature $\kappa$ and the torsion $\tau$ of $\beta$ satisfy the following equation:

$$
\frac{d \tau}{d s}=\frac{\kappa}{\lambda}\left(1+\lambda^{2} \tau^{2}\right)
$$

for some nonzero constant $\lambda$.

Let $M$ be a 3-dimensional non-flat space form, i.e., $\mathbb{S}^{3}$ or $\mathbb{H}^{3}$, and let $\alpha=\alpha(s)$ be a unit speed Frenet curve in $M$ satisfying (2.1) and $\beta=\beta(u)$ a Mannheim partner curve of $\alpha$, where $u$ is the arc length parameter of $\beta$. Then, without loss of generality, the curve $\alpha$ and its principal normal vector field $N_{\alpha}(u)$ can be expressed by

$$
\begin{aligned}
\alpha(u) & =\exp _{\beta(u)}\left(t(u) B_{\beta}(u)\right) \\
& =f(t(u)) \beta(u)+g(t(u)) B_{\beta}(u)
\end{aligned}
$$

and $N_{\alpha}(u)= \pm P^{t(u)}\left(B_{\beta}(u)\right)$, respectively, where the functions $f$ and $g$ are given in (3.4).

Differentiating $\alpha$ with respect to $u$, we have

$$
\begin{aligned}
\dot{\alpha}(u) \frac{d s}{d u}= & \{f(t(u))\}^{\prime} \beta(u)+f(t(u)) T_{\beta}(u) \\
& +\{g(t(u))\}^{\prime} B_{\beta}(u)+g(t(u)) \nabla_{u} B_{\beta}(u) \\
= & \{f(t(u))\}^{\prime} \beta(u)+f(t(u)) T_{\beta}(u) \\
& -\tau_{\beta}(u) g(t(u)) N_{\beta}(u)+\{g(t(u))\}^{\prime} B_{\beta}(u),
\end{aligned}
$$

where $\dot{\alpha}$ denotes the differentiation of $\alpha$ with respect to $s$.

Since $\langle\dot{\alpha}, \alpha\rangle=\left\langle\dot{\alpha}, N_{\alpha}\right\rangle=0$ and $N_{\alpha}=-\epsilon g(t) \beta+f(t) B_{\beta},(3.5)$ shows that $\dot{\alpha}$ is orthogonal to $\beta$ and $B_{\beta}$ in $\mathbb{E}^{4}$ or $\mathbb{E}_{1}^{4}$. Thus, from (4.2), we get

$$
\{f(t(u))\}^{\prime}=\{g(t(u))\}^{\prime}=0 .
$$

Since $t$ is a non-zero smooth function, $t(u)$ is a non-zero constant, say $t(u)=$ $\theta \neq 0$. Then, (4.1) and (4.2) are respectively reduced to

$$
\alpha(u)=f(\theta) \beta(u)+g(\theta) B_{\beta}(u)
$$

and

$$
\dot{\alpha}(u) \frac{d s}{d u}=\left[f(\theta) T_{\beta}(u)-g(\theta) \tau_{\beta}(u) N_{\beta}(u)\right]
$$

from which,

$$
d s / d u=\sqrt{f(\theta)^{2}+\tau_{\beta}(u)^{2} g(\theta)^{2}} .
$$


Hence, we get

$$
T_{\alpha}(u)=b_{1}(u) T_{\beta}(u)+b_{2}(u) N_{\beta}(u),
$$

where $b_{1}(u)=\frac{f(\theta)}{\sqrt{f(\theta)^{2}+\tau_{\beta}(u)^{2} g(\theta)^{2}}}, b_{2}(u)=-\frac{\tau_{\beta}(u) g(\theta)}{\sqrt{f(\theta)^{2}+\tau_{\beta}(u)^{2} g(\theta)^{2}}}$.

Differentiating (4.6) with respect to $u$ gives

$$
\begin{aligned}
\frac{d s}{d u}\left(\nabla_{s} T_{\alpha}(u)-\epsilon \alpha(u)\right)= & -\epsilon b_{1}(u) \beta(u)+\left\{b_{1}^{\prime}(u)-b_{2}(u) \kappa_{\beta}(u)\right\} T_{\beta}(u) \\
& +\left\{b_{1}(u) \kappa_{\beta}(u)+b_{2}^{\prime}(u)\right\} N_{\beta}(u)+b_{2}(u) \tau_{\beta}(u) B_{\beta}(u),
\end{aligned}
$$

from which,

$$
\begin{aligned}
\frac{d s}{d u} \nabla_{s} T_{\alpha}(u)= & \epsilon\left\{f(\theta) \frac{d s}{d u}-b_{1}(u)\right\} \beta(u)+\left\{b_{1}^{\prime}(u)-b_{2}(u) \kappa_{\beta}(u)\right\} T_{\beta}(u) \\
& +\left\{b_{1}(u) \kappa_{\beta}(u)+b_{2}^{\prime}(u)\right\} N_{\beta}(u) \\
& +\left\{b_{2}(u) \tau_{\beta}(u)+\epsilon g(\theta) \frac{d s}{d u}\right\} B_{\beta}(u) .
\end{aligned}
$$

Since $\nabla_{s} T_{\alpha}$ is proportional to $N_{\alpha}=-\epsilon g(\theta) \beta+f(\theta) B_{\beta},(4.7)$ implies that

$$
\left\{\begin{array}{l}
b_{1}^{\prime}(u)-\kappa_{\beta}(u) b_{2}(u)=0, \\
b_{2}^{\prime}(u)+\kappa_{\beta}(u) b_{1}(u)=0
\end{array}\right.
$$

and

$$
\nabla_{s} T_{\alpha}=\epsilon\left\{f(\theta)-b_{1} \frac{d u}{d s}\right\} \beta+\left\{b_{2} \frac{d u}{d s} \tau_{\beta}+\epsilon g(\theta)\right\} B_{\beta} .
$$

By a direct computation, we have

$$
b_{1}^{\prime}-\kappa_{\beta} b_{2}=\frac{g(\theta) \tau_{\beta}\left\{\kappa_{\beta}\left(f(\theta)^{2}+g(\theta)^{2} \tau_{\beta}^{2}\right)-f(\theta) g(\theta) \tau_{\beta}{ }^{\prime}\right\}}{\sqrt{\left[f(\theta)^{2}+g(\theta)^{2} \tau_{\beta}^{2}\right]^{3}}}
$$

and

$$
b_{2}^{\prime}(u)+\kappa_{\beta}(u) b_{1}(u)=\frac{f(\theta)\left\{\kappa_{\beta}\left(f(\theta)^{2}+g(\theta)^{2} \tau_{\beta}^{2}\right)-f(\theta) g(\theta) \tau_{\beta}{ }^{\prime}\right\}}{\sqrt{\left[f(\theta)^{2}+g(\theta)^{2} \tau_{\beta}^{2}\right]^{3}}} .
$$

Thus, equation (4.8) yields

$$
\tau_{\beta}{ }^{\prime}=\frac{\kappa_{\beta}}{\bar{\lambda}}\left\{1+\bar{\lambda}^{2} \tau_{\beta}^{2}\right\},
$$

where $\bar{\lambda}$ is a constant defined by $\bar{\lambda}=\frac{g(\theta)}{f(\theta)}$. Note that $\bar{\lambda}=\theta(\epsilon=0), \tan \theta$ $(\epsilon=1)$ or $\bar{\lambda}=\tanh \theta,|\bar{\lambda}|<1(\epsilon=-1)$.

Conversely, for a curve $\beta$ in $M$ satisfying (4.12) for some $\bar{\lambda}(\bar{\lambda} \neq 0$ if $\epsilon=1$, $|\bar{\lambda}|<1$ if $\epsilon=-1$ ), we may choose $\theta \in \mathbb{R}$ such that $\bar{\lambda}=\frac{g(\theta)}{f(\theta)}$. We define a curve $\alpha$ by (4.4). Then, from (4.10) and (4.11), (4.7) implies (4.9). 
When $M=\mathbb{S}^{3}$, (4.9) becomes

$$
\nabla_{s} T_{\alpha}=\frac{\sin \theta \cos \theta\left(\tau_{\beta}^{2}-1\right)}{\cos ^{2} \theta+\sin ^{2} \theta \tau_{\beta}{ }^{2}}\left\{\sin \theta \beta-\cos \theta B_{\beta}\right\},
$$

from which,

$$
\left\|\nabla_{s} T_{\alpha}\right\|=\frac{\left|\sin 2 \theta\left(\tau_{\beta}^{2}-1\right)\right|}{2\left(\cos \theta^{2}+\sin \theta^{2} \tau_{\beta}{ }^{2}\right)} .
$$

This means that if $\sin 2 \theta\left(\tau_{\beta}^{2}-1\right)>0, \beta$ is a Mannheim partner curve of $\alpha$. In fact, for a function $\tau_{\beta} \neq \pm 1$, we can choose locally a constant $\theta$ satisfying $\sin 2 \theta\left(\tau_{\beta}^{2}-1\right)>0$. Thus, the principal normal vector field $N_{\alpha}$ of $\alpha$ is given by

$$
N_{\alpha}(u)=-\sin \theta \beta(u)+\cos \theta B_{\beta}(u) .
$$

By the similar method, when $M=\mathbb{H}^{3}, \beta$ is a Mannheim partner curve of $\alpha$.

Consequently, we have a characterization of Mannheim partner curves in a 3-dimensional space form.

Theorem 4.2. Let $\beta=\beta(s)$ be a Frenet curve in a 3-dimensional space form $M$ with the arc parameter s. Then, $\beta$ is the Mannheim partner curve of some Mannheim curve $\alpha$ in $M$ if and only if the curvature $\kappa$ and the torsion $\tau$ of $\beta$ satisfy (4.12) for a constant $\bar{\lambda}\left(\bar{\lambda} \neq 0\right.$ if $M=\mathbb{E}^{3}$ or $\mathbb{S}^{3},|\bar{\lambda}|<1$ if $\left.M=\mathbb{H}^{3}\right)$ with (3.4).

A helix in a 3-dimensional manifold $M$ is defined by a curve whose curvature and torison are constants. In [2], Huili Liu and Fan Wang stated that the Mannheim partner curve of a helix in $\mathbb{E}^{3}$ is a straight line. Motivated by this result, we give the following example:

Example. Let $M$ be a 3-dimensional non-flat space form, i.e., $M=\mathbb{S}^{3}$ or $M=\mathbb{H}^{3}$, and $\alpha=\alpha(s)$ a helix in $M$ parametrized by the arc length with the curvature $\kappa_{0}$ and the torsion $\tau_{0}$.

By applying $\kappa_{0}$ and $\tau_{0}$ to (3.18), we have $\lambda=\frac{\kappa_{0}^{2}+\tau_{0}^{2}-1}{\kappa_{0}}$ and $\lambda=\frac{\kappa_{0}^{2}+\tau_{0}^{2}+1}{\kappa_{0}}$ according to $M=\mathbb{S}^{3}$ and $M=\mathbb{H}^{3}$, respectively. This means that $\alpha$ is a Mannheim curve in $M$. Moreover, the Mannheim partner curve $\beta$ of $\alpha$ in $\mathbb{S}^{3}$ $\left(\right.$ resp. $\left.\mathbb{H}^{3}\right)$ is given by $\beta(u)=\cos \theta \alpha(u)+\sin \theta N_{\alpha}(u)($ resp. $\beta(u)=\cosh \theta \alpha(u)+$ $\left.\sinh \theta N_{\alpha}(u)\right)$ with

$$
\begin{aligned}
& u=\sqrt{\left(\cos \theta-\kappa_{0} \sin \theta\right)^{2}+\tau_{0}^{2} \sin ^{2} \theta} s\left(\text { resp. } \sqrt{\left(\cosh \theta-\kappa_{0} \sinh \theta\right)^{2}+\tau_{0}^{2} \sinh ^{2} \theta} s\right), \\
& \theta=\frac{1}{2} \tan ^{-1}\left(\frac{2 \kappa_{0}}{\kappa_{0}^{2}+\tau_{0}^{2}-1}\right)\left(\text { resp. } \frac{1}{2} \tanh ^{-1}\left(\frac{2 \kappa_{0}}{\kappa_{0}^{2}+\tau_{0}^{2}+1}\right)\right),
\end{aligned}
$$

where $N_{\alpha}$ is the principal normal vector field of $\alpha$ and $u$ is the arc length parameter of $\beta$.

On the other hand, equations (3.10), (3.14) and (3.15) lead to $\nabla_{u} T_{\beta}=0$, from which, $\beta$ is a geodesic in $M$.

Consequently, we have the following proposition: 
Proposition 4.3. A helix in a 3-dimensional simply connected space form $M$ is a Mannheim curve. Moreover, the Mannheim partner curve of a helix in $M$ is a geodesic.

\section{References}

[1] J. H. Choi, T. H. Kang, and Y. H. Kim, Bertrand curves in 3-dimensional space forms, to appear in Applied Mathematics and Computation.

[2] H. Liu and F. Wang, Mannheim partner curves in 3-space, J. Geom. 88 (2008), no. 1-2, $120-126$.

Jin Ho CHOI

Department of Mathematics

UNIVERSITY OF ULSAN

ULSAN 680-749, KOREA

E-mail address: jino7142@knu.ac.kr

TAe Ho Kang

Department of Mathematics

UNIVERSITY OF ULSAN

UlSAN 680-749, KoreA

E-mail address: thkang@ulsan.ac.kr

Young Ho Kim

Department of Mathematics

KyUngpook National University

TAEgu 702-701, Korea

E-mail address: yhkim@knu.ac.kr 